\title{
Anti-chondroitin sulfate proteoglycan 4-specific antibodies modify the effects of vemurafenib on melanoma cells differentially in normoxia and hypoxia
}

\author{
DANIELA PUCCIARELLI ${ }^{1}$, NINA LENGGER ${ }^{1}$, MARTINA TAKACOVA $^{2}$, LUCIA CSADEROVA $^{2}$, \\ MARIA BARTOSOVA $^{2}$, HEIMO BREITENEDER ${ }^{1}$, SILVIA PASTOREKOVA $^{2}$ and CHRISTINE HAFNER ${ }^{1,3}$ \\ ${ }^{1}$ Department of Pathophysiology and Allergy Research, Center for Pathophysiology, Infectiology and Immunology, \\ Medical University of Vienna, Vienna, Austria; ${ }^{2}$ Institute of Virology, Department of Molecular Medicine, Slovak Academy \\ of Sciences, Bratislava, Slovakia; ${ }^{3}$ Karl Landsteiner Institute for Dermatological Research, St. Poelten, Austria
}

Received March 5, 2015; Accepted April 16, 2015

DOI: 10.3892/ijo.2015.3010

\begin{abstract}
Chondroitin sulfate proteoglycan 4 (CSPG4), a highly immunogenic melanoma tumor antigen, is a potential target for antibody-based immunotherapy. The mechanism by which CSPG4 affects melanoma progression is only partly understood, in particular the involvement of other receptor tyrosine kinases and the tumor microenvironment. We have previously reported on a mimotope-based vaccine against CSPG4 in a human melanoma xenograft model that resulted in reduction of tumor growth. Herein we describe the influence of hypoxia on the response to polyclonal antiCSPG4-antibodies induced by this vaccine in combination with the BRAF inhibitor vemurafenib to enhance therapeutic efficacy by simultaneously targeting multiple signaling pathways. Melanoma cells were treated with polyclonal antiCSPG4-antibodies and vemurafenib. Proliferation, migration and invasion were evaluated in a real-time setting in the impedance-based x-CELLigence ${ }^{\circledR}$ system. Western blotting and quantitative PCR arrays were used to determine protein and mRNA expression of hypoxia inducible factor $1 \alpha(\mathrm{HIF} 1 \alpha)$, carbonic anhydrase IX (CAIX) and signaling pathway proteins. A melanoma xenograft model was used to detect HIF1 $\alpha$ and CAIX expression in vivo. Hypoxia enhanced the antiproliferative response to vemurafenib. The migration and
\end{abstract}

Correspondence to: Dr Christine Hafner, Department of Pathophysiology and Allergy Research, Center for Pathophysiology, Infectiology and Immunology, Medical University of Vienna, Waehringer Guertel 18-20, 1090 Vienna, Austria

E-mail: christine.hafner@meduniwien.ac.at

Abbreviations: CSPG4, chondroitin sulfate proteoglycan 4; HIF1 $\alpha$, hypoxia-inducible factor $1 \alpha$; CAIX, carbonic anhydrase IX; DMOG, dimethyloxalylglycine, N-(methoxyoxoacetyl)-glycine methyl ester; PVDF, polyvinylidene fluoride

Key words: melanoma, chondroitin sulfate proteoglycan 4, hypoxia, vemurafenib, hypoxia-inducible factor $1 \alpha$ invasion capacities of vemurafenib-treated melanoma cells were increased, in spite of vemurafenib-decreased expression of HIF1 $\alpha$ and CAIX. Polyclonal anti-CSPG4-antibodies reduced the Transwell migration of vemurafenib-treated, BRAF V600E-mutant and CSPG4-expressing melanoma cells in hypoxia. This was associated with the downregulation of phosphorylated AKT, a kinase contributing to tumor cell migration. Our results highlight CSPG4 as a potential target for modulating treatment resistance to vemurafenib induced by the hypoxic microenvironment.

\section{Introduction}

The incidence of melanoma has increased over the past decades and this tumor entity is responsible for the majority of skin cancer-related deaths $(1,2)$. Melanoma is one of the most challenging types of cancer to be treated due to its high heterogeneity. Surgery remains the main treatment option for early-stage melanomas (3). However, the prognosis of patients diagnosed with metastatic melanoma is still poor, with only a limited number of agents available for treatment $(4,5)$. One of the latest successful drugs for the treatment of advanced melanoma is the BRAF inhibitor vemurafenib (also known as PLX4032) that showed an increase in overall survival and an extension of progression-free survival $(6,7)$. However, incomplete response and acquired drug resistance caused by multiple abnormally regulated signaling pathways present in melanoma frequently led to treatment failures and tumor progression (8-10). Therefore, simultaneously targeting multiple signaling pathways has great potential for enhancing therapeutic efficacy and overcoming resistance to vemurafenib $(10,11)$.

The chondroitin sulfate proteoglycan 4 (CSPG4) is a cellsurface proteoglycan involved in the activation of several signaling pathways playing an important role in tumor cell proliferation, survival, and migration as well as in tumor progression (12). Since it is highly expressed on tumor cells and has restricted distribution in normal tissues, CSPG4 has been used as a target of antibody-based immunotherapy $(13,14)$. The CSPG4-specific monoclonal antibody 225.28 was shown to induce regression of tumor metastases, to inhibit spontaneous 
metastasis and tumor recurrence in breast cancer mouse models (15), and to inhibit the growth and recurrence of melanoma in a human melanoma xenograft model (16). Recent findings have shown that the combination of vemurafenib and the CSPG4-specific mAb 225.28 was capable of blocking the FAK and PKC $\alpha$ signaling pathways which are important for cell growth, migration, and survival (17). Antibodies induced by a mimotope vaccine (anti-225D9+-TT Abs) against the epitope defined by the mAb 225.28 were capable of inhibiting melanoma cell growth in vitro and in vivo $(18,19)$.

As many other cancers, melanomas include regions of hypoxia caused by an imbalance between oxygen supply and consumption. The response to treatment is affected by this microenvironmental factor $(20,21)$. Tumor hypoxia can negatively influence treatment outcome and patient survival in various cancer types $(22,23)$. Melanomas appear to downregulate signaling pathways associated with proliferation in order to migrate (24). Hypoxia has been shown to enhance the cell migratory propensity and invasiveness and to contribute to cancer metastasis (25) through the hypoxia inducible factor $1 \alpha(\mathrm{HIF} 1 \alpha)$. HIF1 $\alpha$ regulates genes that are regarded pro-tumorigenic $(26,27)$ and increases the expression of a number of genes involved in invasion (28). Carbonic anhydrase IX (CAIX), a direct transcriptional target of HIF1 $\alpha$, plays an important role in maintaining $\mathrm{pH}$ homeostasis (29). Earlier studies have shown that a BRAF V600E mutation increased HIF1 $\alpha$ expression under hypoxic conditions (30). Hypoxia also induced phenotypic plasticity and therapy resistance in melanoma cells via tyrosine kinase receptors (21).

Herein we report on the response of CSPG4-specific anti$225 \mathrm{D} 9^{+}-\mathrm{TT}$ Abs to enhance the anti-proliferative effects of vemurafenib in normoxia. We also describe the role of hypoxia on the response to vemurafenib and anti-225D9+-TT Abs and its effect on the anti-proliferative, migratory and invasive potential of melanoma cells.

\section{Materials and methods}

Cell lines, BRAF inhibitor and polyclonal antibodies. The human CSPG4 expressing $\left(\mathrm{CSPG}^{+}\right)$melanoma cell line 518A2 and the human CSPG4 negative (CSPG4) melanoma cell line M14, both harboring the V600E BRAF mutation, were described elsewhere $(17,18)$. Routine tests to exclude mycoplasma and characterize the origin of the cells (short tandem repeat analysis) were performed. Vemurafenib (PLX4032, Selleckchem, Houston, TX, USA) is a potent selective inhibitor of BRAFV600. Polyclonal anti-225D9+-TT Abs recognizing CSPG4 were developed and characterized as previously described $(18,19)$. Isotype control anti-TT Abs were used as negative control. Exposure to hypoxia was performed in an anaerobic work station (Ruskin Technologies, Bridgend, $\mathrm{UK}$ ) in $2 \% \mathrm{O}_{2}, 5 \% \mathrm{CO}_{2}, 10 \% \mathrm{H}_{2}$, and $83 \% \mathrm{~N}_{2}$ at $37^{\circ} \mathrm{C}$.

Cell proliferation assays in normoxic and hypoxic conditions. The impedance-based $\mathrm{x}$-CELLingence system (ACEA Bioscience Inc., San Diego, CA, USA) was placed at $37^{\circ} \mathrm{C}$ in a humidified $5 \% \mathrm{CO}_{2}$ incubator. 518A 2 cells $\left(5 \times 10^{3}\right)$ were seeded in each well and placed in the $\mathrm{x}$-CELLingence system and proliferation was measured for $24 \mathrm{~h}$. After $24 \mathrm{~h}$ the following compounds were added: i) $5 \mu \mathrm{M}$ vemurafenib, ii) $1 \mathrm{mM}$ DMOG [dimethyloxalylglycine, N-(methoxyoxoacetyl)-glycine methyl ester] (Sigma-Aldrich, St. Louis, MO, USA), and iii) $5 \mu \mathrm{M}$ vemurafenib plus $1 \mathrm{mM}$ DMOG. The plate was placed back in the $\mathrm{x}$-CELLingence system and measured for $100 \mathrm{~h}$. DMOG was used to induce hypoxia in cells when it was technically not possible to use a hypoxia chamber.

Cell proliferation with anti-225D9+-TT Abs and vemurafenib. In order to determine the optimal doses of vemurafenib on 518A 2 and M14 melanoma cell lines, dose-titration experiments were performed. 518A2 (CSPG4 $\left.{ }^{+}\right)$and M14 (CSPG4) cells $\left(2 \times 10^{3}\right)$ per well were seeded and vemurafenib was added at different concentrations $(0.001,0.01,0.1,0.5,1.0,10.0 \mu \mathrm{M})$. A $\left[{ }^{3} \mathrm{H}\right]$-Thymidine incorporation assay was performed and percentage of inhibition of proliferation was calculated by comparing the CPM values of treated cells with those of untreated cells, which were set at $100 \%$.

To test the combinatorial treatment of vemurafenib and anti225D9+-TT Abs $\left(2 \times 10^{3}\right)$ 518A2 $\left(\mathrm{CSPG}^{+}\right)$and M14 (CSPG4-) cells per well were seeded and incubated with anti-225D9+-TT Abs or isotype control anti-TT Abs at a concentration of $200 \mu \mathrm{g} / \mathrm{ml}$. A $\left[{ }^{3} \mathrm{H}\right]$-Thymidine incorporation assay was performed as previously described (18). To test the long-term effect of vemurafenib combined with anti-225D9+-TT Abs, an 8-day proliferation assay was performed. 518A2 $\left(\mathrm{CSPG}^{+}\right)$ and M14 (CSPG4 $\left.{ }^{-}\right)$cells $\left(2 \times 10^{3}\right)$ were seeded. After $24 \mathrm{~h}$, cells were treated with $1 \mu \mathrm{M}$ vemurafenib, $1 \mu \mathrm{M}$ vemurafenib plus $200 \mu \mathrm{g} / \mathrm{ml}$ anti-225D9 ${ }^{+}-\mathrm{TT}$ Abs, or $1 \mu \mathrm{M}$ vemurafenib plus $200 \mu \mathrm{g} / \mathrm{ml}$ isotype control TT Abs. On day 4, 6 and 8 a $\left[{ }^{3} \mathrm{H}\right]$-Thymidine incorporation assay was performed.

Transwell migration and Transwell invasion assays. The CIM-Plate $16(8-\mu \mathrm{m}$ pore diameter; ACEA Bioscience Inc.) with or without matrigel $[1 / 10$ in medium with $10 \%$ FCS (BD Biosciences, Franklin Lakes, NJ, USA)], was used for Transwell invasion or migration assays (31). 518A2 cells $\left(4 \times 10^{5}\right)$ were seeded in quadruplicates and treated with i) serum-free medium (SFM), ii) medium supplemented with $10 \%$ fetal calf serum (FSC), iii) vemurafenib $(1 \mu \mathrm{M})$, and iv) vemurafenib $(1 \mu \mathrm{M})$ plus anti-225D9 ${ }^{+}-\mathrm{TT}$ Abs $(200 \mu \mathrm{g} / \mathrm{ml})$. The plates were placed in the RTCA DP Analyzer (ACEA Bioscience Inc.) in normoxia or hypoxia and numbers of migrated or invaded cells measured for $48 \mathrm{~h}$. Cell index values are directly proportional to the measured impedances which are automatically and continuously recorded by the RTCA DP instrument.

Determination of extracellular $\mathrm{pH}$ and oxygen consumption in melanoma cells. Since extracellular $\mathrm{pH}$ regulation and oxygen consumption are affected by hypoxia, which induces a cell-type specific shift in glycolysis and thereby alters the consumption of oxygen (32), a SDR optical sensor system (1450 MicroBeta TriLux; Perkin-Elmer, Waltham, MA, USA) embedded in the hypoxic box was used for measuring these two parameters. 518A2 (CSPG4 ${ }^{+}$) and M14 (CSPG4) cells $\left(0.2 \times 10^{6}\right)$ were seeded and incubated with i) $1 \mu \mathrm{M}$ vemurafenib, ii) $1 \mu \mathrm{M}$ vemurafenib plus $200 \mu \mathrm{g} / \mathrm{ml}$ anti-225D9+-TT Abs, and iii) $200 \mu \mathrm{g} / \mathrm{ml}$ anti-225D9+-TT Abs. The plates were placed in the hypoxia chamber with $2 \%$ of atmospheric oxygen and the extracellular $\mathrm{pH}$ and oxygen consumption were measured by using the SDR optical sensor system. The sensor 
dish reader monitors the $\mathrm{pH}$ in real-time in an OxoDish ${ }^{\circledR}$ for oxygen and in a HydroDish ${ }^{\circledR}$ for $\mathrm{pH}$ using a non-invasive technique that detects the luminescence lifetime of a sensor spot at the bottom of each well that is dependent on the $\mathrm{pH}$ of the surrounding sample. The extracellular $\mathrm{pH}$ was measured by the SDR every $25 \mathrm{~min}$ for $112 \mathrm{~h}$ and the oxygen consumption was measured by the SDR every $5 \mathrm{~min}$ for $5 \mathrm{~h}$.

$R N A$ extraction and quantitative reverse transcription-PCR. Total RNA was extracted with the TRIzol reagent (SigmaAldrich) from cells that had been treated with vemurafenib $(1 \mu \mathrm{M})$ and cultured in hypoxic or normoxic conditions for $24 \mathrm{~h}$. Three micrograms of RNA were reverse-transcribed into cDNA with High-Capacity cDNA Reverse Transcription kit (Applied Biosystems, Foster City, CA, USA) using random heptameric primers. Quantitative RT-PCR analysis of mRNAs of HIFl $\alpha, C A 9$ and $\beta$-actin as internal standard were performed on a StepOne ${ }^{\mathrm{TM}}$ Real-time PCR System (Applied Biosystems) using Power SYBR ${ }^{\circledR}$ Green PCR Master mix (Applied Biosystems). The following primers were used: HIFl $\alpha$ sense: 5'-GCTTGGTGCTGATTTGTGAACC-3', HIFl $\alpha$ antisense: 5'-GCATCCTGTACTGTCCTGTGGTG-3'; CA9 sense: 5'-CCGAGCGACGCAGCCTTTGA-3', CA9 antisense: 5'-GGCTCCAGTCTCGGCTACCT-3'; $\beta$-actin sense: 5'-TCC TCCCTGGAGAAGAGCTA-3', $\beta$-actin antisense: 5'-ACATC TGCTGGAAGGTGGAC-3'. The results were analyzed using the Applied Biosystems 7500 system v1.4.0 software.

Immunoblot analysis. 518A2 melanoma cells were incubated with vemurafenib $(1 \mu \mathrm{M})$, anti-225D9+-TT Abs $(200 \mu \mathrm{g} / \mathrm{ml})$ or vemurafenib $(1 \mu \mathrm{M})$ plus anti-225D9 ${ }^{+}-\mathrm{TT}$ Abs $(200 \mu \mathrm{g} / \mathrm{ml})$ and placed in normoxia or hypoxia for $24 \mathrm{~h}$. After treatment cells were lysed and equal amounts of proteins were separated by SDS-PAGE under reducing conditions and transferred onto polyvinylidene fluoride (PVDF) membranes (Immobilon Millipore, Billerica, MA, USA). Cells were lysed in lysis buffer [1\% Triton $\mathrm{X}-100,150 \mathrm{mM} \mathrm{NaCl}, 50 \mathrm{mM}$ Tris, $\mathrm{pH} 7.5$, $0.5 \%$ Nonidet P-40, $50 \mathrm{mM} \mathrm{NaF}$, protease inhibitor cocktail (Roche, Mannheim, Germany) or $10 \mathrm{mM}$ Tris/HCl pH 8.2, $1 \%$ NP40, 1 mM EDTA, $150 \mathrm{mM} \mathrm{NaCl}$, protease inhibitor cocktail (Roche)]. Primary antibodies used recognized human CAIX [M75, (33)], HIF1 $\alpha$ (BD Transduction Laboratories, San Jose, CA, USA), pFAK(Tyr397), FAK, PKC $\alpha$ (Abcam, Cambridge, UK) and pAKT (Ser473), AKT, pERK 1/2(Thr202/Tyr204), ERK1/2 or $\beta$-actin mAbs (Cell Signaling Technology, Danvers, MA, USA). Corresponding peroxidase-conjugated secondary mAbs (Cell Signaling Technology) were used. Blots were developed using the LumiGLO chemiluminescencent substrate (Cell Signaling Technology) and bands visualized using the Filmentwickler CP1000 Processor (AGFA, Mortsel, Belgium).

Immunohistochemistry in xenografts. The human melanoma C.B.17 SCID/SCID mouse xenotransplantation model was described elsewhere (16). All experiments were approved by the Animal Experimentation Committee of the University of Vienna and the Ministry of Education, Science and Culture, Vienna, Austria. Untreated 518A2 melanoma tumors were fixed in $4 \%$ paraformaldehyde and embedded in paraffin. For CAIX detection, sections were stained in the Dako Autostainer using the DakoCytomation EnVision ${ }^{\circledR}+$ System-HRP (Dako, Glostrup, Denmark) according to the manufacturer's instructions. Antibodies used were the anti-CAIX antibody M75 and a secondary anti-mouse IgG antibody (Dako). Staining was visualized with DAB solution (Dako). For HIF1 $\alpha$ immunostaining, antigen retrieval was carried out with citrate buffer, $\mathrm{pH} 6.0$, for $5 \mathrm{~min}$ at $125^{\circ} \mathrm{C}$ using a Pascal pressure chamber (Dako). Deparaffinized sections were stained with DakoCytomation Catalysed Signal Amplification System according to the manufacturer's instructions. Sections were incubated with a primary antibody specific for HIF1 $\alpha$ (1:250; BD Transduction Laboratories). Staining was visualized with DAB solution. The stained sections were examined with an Olympus BX53 microscope and photographed with an Olympus DP73 camera (Olympus Electronics, Tokyo, Japan).

\section{Results}

Vemurafenib inhibits cell growth of BRAF V600E mutant $518 A 2$ melanoma cells in normoxic and hypoxic conditions. Treatment of melanoma cells with increasing concentrations of vemurafenib resulted in a dose-dependent inhibition of proliferation of the BRAF V600E mutant melanoma cell lines 518A2 and M14. The growth of the BRAF V600E mutant 518A2 melanoma cells and M14 melanoma cells treated with $1 \mu \mathrm{M}$ vemurafenib was inhibited by $40 \pm 3 \%$ and $60 \pm 5 \%$, respectively (data not shown). In order to visualize the timecourse of the inhibitory effect of vemurafenib, a real-time characterization was performed using the $\mathrm{x}$-CELLingence system. 518A 2 cells were treated with $5 \mu \mathrm{M}$ vemurafenib and cell proliferation was measured for up to $130 \mathrm{~h}$ in normoxic and hypoxic conditions. 518A2 cells were not susceptible to hypoxic conditions and the proliferation rate did not change (maximum cell index of 5.3). After addition of vemurafenib, hypoxic 518A2 cells (maximum cell index of 2.8) reduced cell growth by an additional $38 \%$ compared to normoxic, vemurafenib-treated 518A2 cells (maximum cell index of 4.3) (Fig. 1A). The inhibitory peak after vemurafenib in normoxic conditions was reached after $60 \mathrm{~h}$, whereas hypoxia shifted the peak to $90 \mathrm{~h}$ (Fig. 1A).

Effect of hypoxia on HIFl $\alpha$ and CAIX protein expression and mRNA levels in vemurafenib-treated 518 A2 melanoma cells. To investigate how hypoxia influences the response of 518A2 cells to vemurafenib, we focused on important markers of hypoxia, HIF1 $\alpha$ and CAIX. Messenger RNA levels of HIFl $\alpha$ did not show significant changes. The relative mRNA level of HIF1 $\alpha$ in $518 \mathrm{~A} 2$ cells in hypoxia was $0.8 \pm 0.08$. Treatment with vemurafenib increased the mRNA level of $H I F l \alpha$ to $0.9 \pm 0.02$. In normoxic conditions the relative mRNA levels of $H I F l \alpha$ were $1.0 \pm 0.1$ and $0.8 \pm 0.17$ for untreated and treated 518A 2 cells, respectively (Fig. 1B). The relative CA9 mRNA levels were $1.0 \pm 0.5$ in normoxic conditions with and without vemurafenib treatment. In hypoxic conditions $C A 9$ mRNA levels increased to $12.0 \pm 0.9$ for untreated cells and to $16.0 \pm 1.1$ for vemurafenib-treated cells (Fig. 1B). All mRNA levels have been normalized to the housekeeping gene coding for $\beta$-actin. Both proteins were expressed in hypoxic 518A 2 cells. After treatment with vemurafenib in hypoxic conditions HIF1 $\alpha$ and CAIX protein expression was downregulated, whereas in 

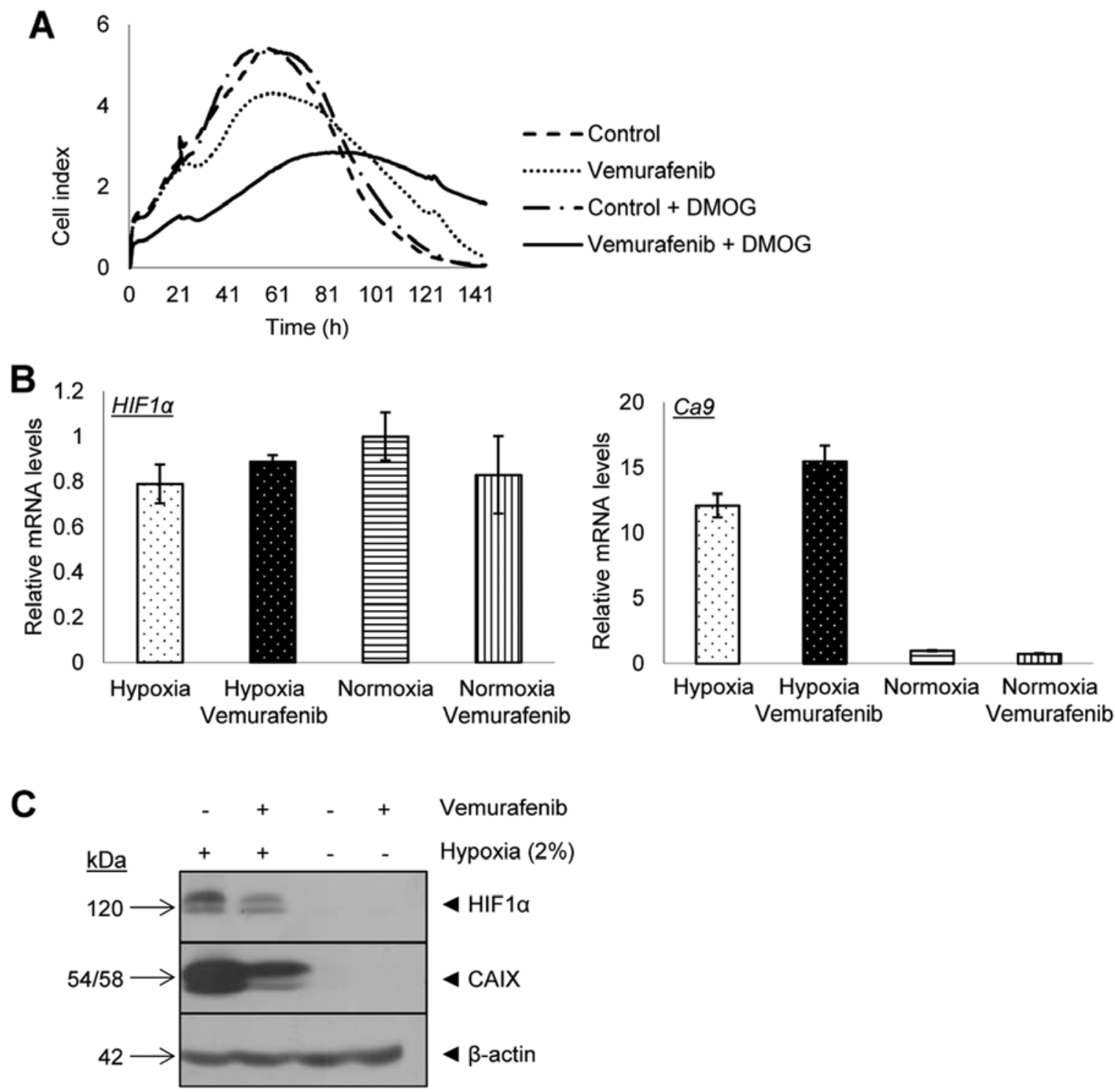

Figure 1. Hypoxia influences the response of vemurafenib in 518A2 melanoma cells and effects mRNA levels and protein expression of HIF1 $\alpha$ and CAIX. (A) Time lapse measurements of the inhibitory effect of vemurafenib in normoxic and hypoxic conditions by the x-CELLigence system. (B) Real-time PCR analysis of HIFl $\alpha$ and CA9 mRNA levels, normalized to the internal control ( $\beta$-actin). Error bars represent \pm SD from three different experiments. (C) Expression of HIF1 $\alpha$ and CAIX proteins in vemurafenib-treated 518A2 cells was evaluated by immunoblot analyses.

normoxic conditions HIF1 $\alpha$ and CAIX were not expressed in either treated or untreated 518A2 cells (Fig. 1C).

Expression of hypoxia markers in melanoma xenografts. The expression of HIF1 $\alpha$ and CAIX in a 518A2 melanoma xenograft model was evaluated by immunohistochemistry. As shown in Fig. 2, immunohistochemical staining of formalinfixed, paraffin-embedded tumor tissues demonstrated the presence of HIF1 $\alpha$ and CAIX, which showed typical, hypoxiarelated expression patterns. These in vivo results reflect the expression level of both proteins observed in vitro.

Anti-225D9+-TT Abs enhance the effects of vemurafenib in CSPG4 expressing 518A2 melanoma cells. Targeting multiple signaling pathways was more effective in suppressing the growth of BRAF V600E mutant melanoma cells (11). Treatment with CSPG4 specific anti-225D9+-TT Abs showed that the growth of 518A2 cells [BRAF(V600E)/CSPG4 ${ }^{+}$] was inhibited by $32 \pm 5.9 \%$ compared to M14 cells [BRAF(V600E)/ CSPG4-] (Fig. 3A). When combining vemurafenib with anti225D9+-TT Abs, this led to an additional growth inhibition of the $518 \mathrm{~A} 2$ [BRAF(V600E)/CSPG4 ${ }^{+}$] by $30 \pm 6.0 \%$ on day 8 compared to the CSPG4 negative cell line M14 (Fig. 3B). These results showed that targeting the CSPG4 protein alone and in combination with vemurafenib influenced the proliferation rate of 518A2 melanoma cells.

Influence of vemurafenib and anti-225D9+-TT Abs on the Transwell migration and Transwell invasion capacity of 518 A2 melanoma cells. In order to measure the tumorigenicity of the 518A2 melanoma cells in hypoxic and normoxic conditions, a Transwell migration assay using the RTCA DP Analyzer was performed (Fig. 4A). In normoxic conditions, untreated 518A2 cells migrated relatively slowly but continuously and reached a cell index of 3.3 after $50 \mathrm{~h}$. In the presence of vemurafenib, the cells initially migrated and reached a cell index of 1.8 after 

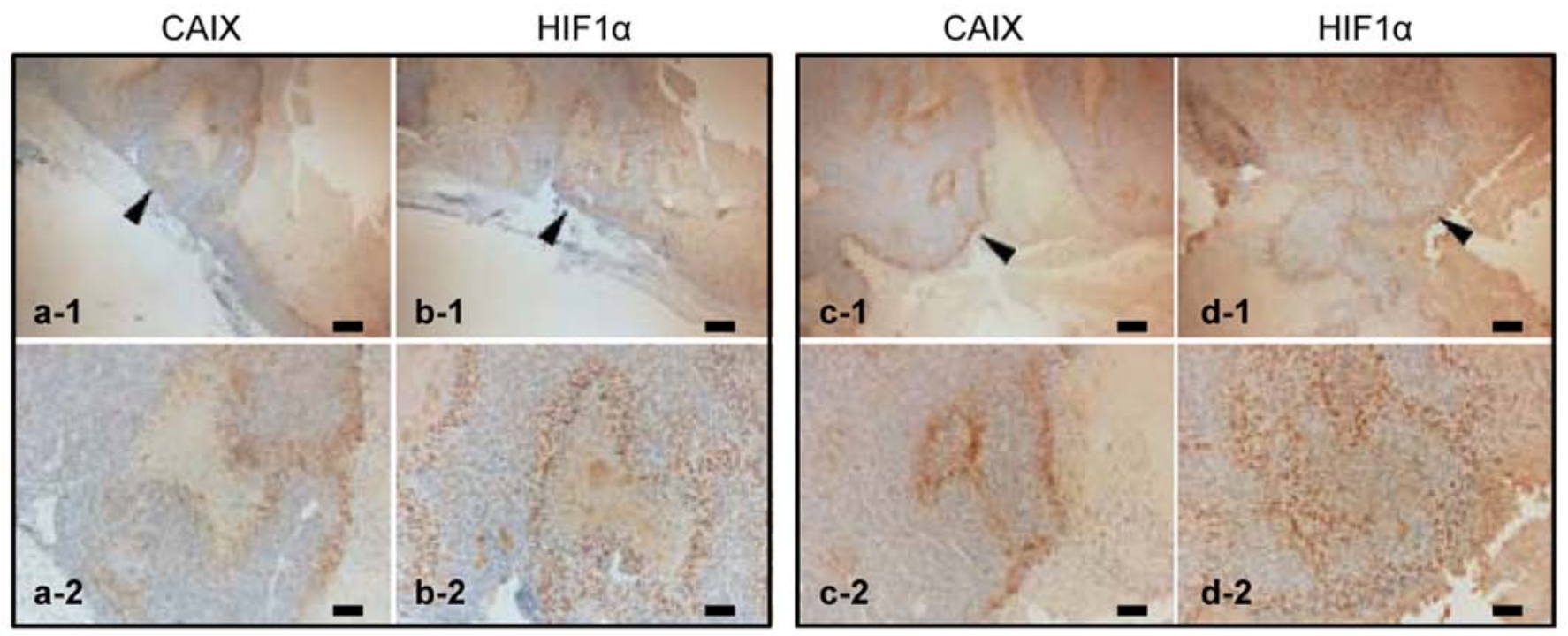

Figure 2. Immunohistochemistry of two representative 518A2 xenograft tumor samples stained with HIF1 $\alpha$ and CAIX antibodies from corresponding tumor regions. Overview images of two representative melanoma tumors are shown in the top row (a-1, b-1 and c-1, d-1); staining for CAIX (a-1, a-2, c-1 and c-2) and HIF1 $\alpha$ (b-1, b-2, d-1 and d-2) shows regions with high expression. Scale bars, $200 \mu \mathrm{m}$ (a-1, b-1, c-1 and d-1), and $100 \mu \mathrm{m}$ (a-2, b-2, c-2 and d-2). Arrows point to the area of magnification.
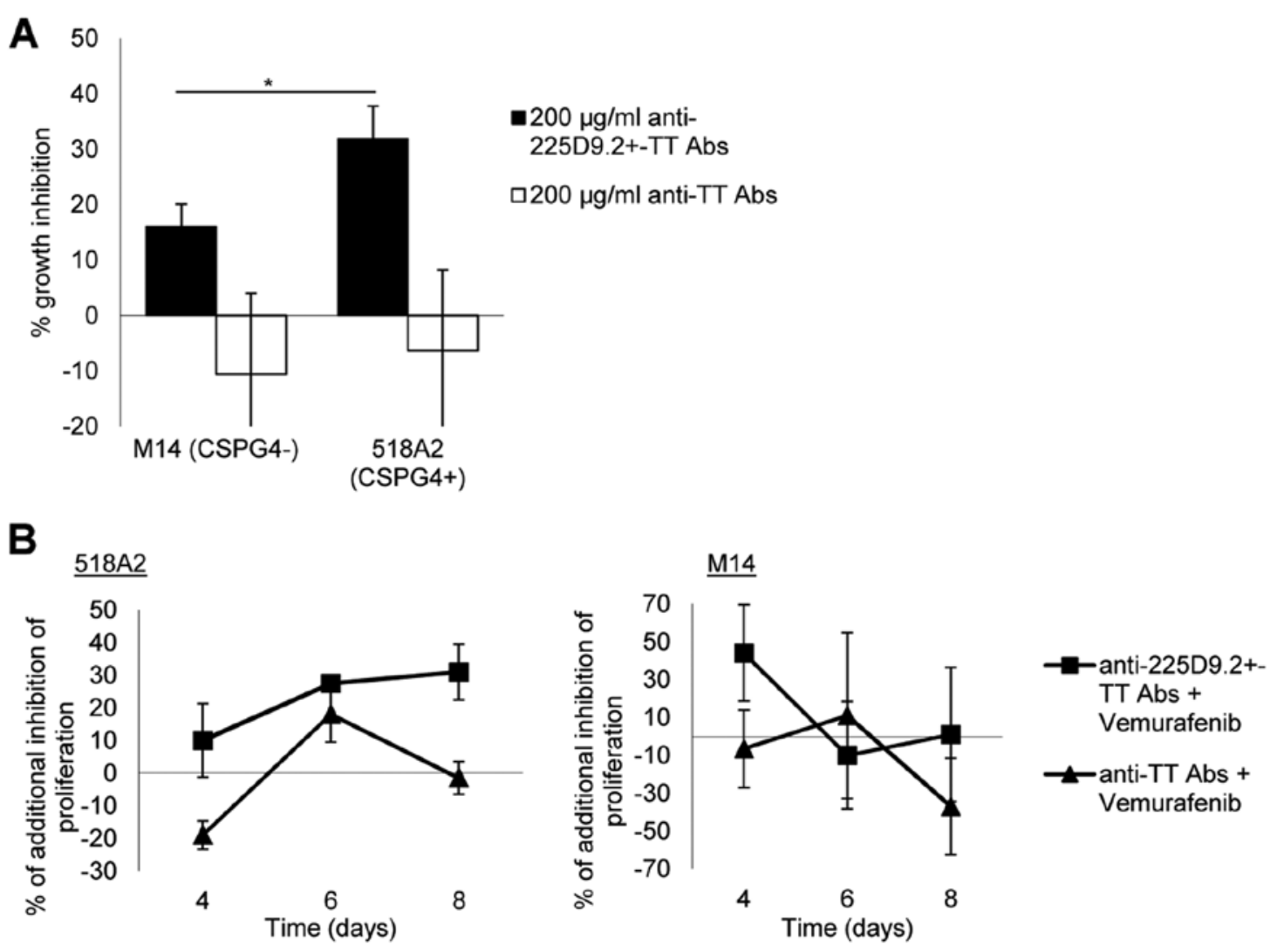

Figure 3. CSPG4-specific anti-225D9+-TT Abs enhance the antiproliferative effects of vemurafenib in 518A2 melanoma cells. (A) Growth inhibition of 518A2 melanoma cells [BRAF(V600E)/CSPG4 ${ }^{+}$] by anti-225D9+-TT Abs compared to M14 melanoma cells [BRAF(V600E)/CSPG4] ("P<0.05). (B) Anti-225D9 ${ }^{+}$-TT Abs contribute to an additional growth inhibition in vemurafenib-treated 518A2 melanoma cells [BRAF(V600E)/CSPG4 $4^{+}$which was not the case in M14 melanoma cells [BRAF(V600E)/CSPG4- ]. Anti-TT Abs were used as isotype control. Error bars represent \pm SD from three different experiments.

$15 \mathrm{~h}$, and then their migration index decreased to 0.9 after $38 \mathrm{~h}$. The combination of vemurafenib and anti-225D9+-TT Abs had a similar effect on the course of migration, since the cell index initially increased to 2.6 after $22 \mathrm{~h}$ and then decreased to 1.6 after $46 \mathrm{~h}$. As expected, in hypoxic conditions the cell index was markedly increased in untreated 518A2 melanoma cells, where it reached a value of 5.0 after $52 \mathrm{~h}$. The migration capacity of vemurafenib treated melanoma cells was 

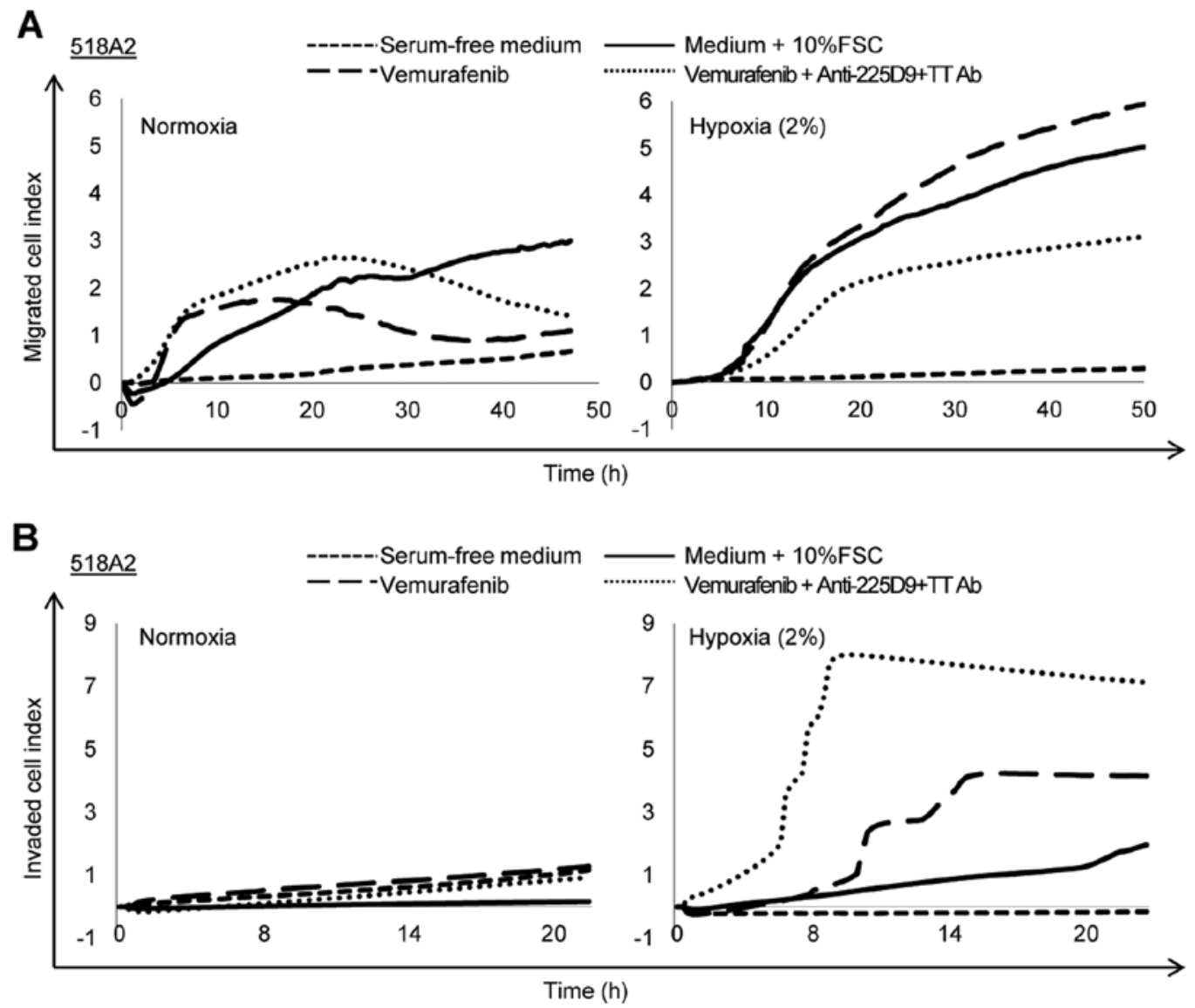

Figure 4. CSPG4-specific anti-225D9+-TT Abs decrease Transwell migration but increase Transwell invasion in hypoxic, vemurafenib-treated melanoma cells. (A) Migration capacity of anti-225D9+-TT Abs- and vemurafenib-treated 518A2 melanoma cells in normoxic and hypoxic conditions. (B) Invasion capacity of anti-225D9+-TT Abs- and vemurafenib-treated 518A2 melanoma cells in normoxic and hypoxic conditions.

Table I. Extracellular $\mathrm{pH}$ measured $75 \mathrm{~h}$ after the administration of vemurafenib and anti-225D9+-TT Abs to hypoxic 518A2 melanoma cells [BRAF(V600E)/CSPG4 ${ }^{+}$and hypoxic M14 melanoma cells [BRAF(V600E)/CSPG4]

\begin{tabular}{lcccc} 
Cells & Control & Vemurafenib & Anti-225D9 ${ }^{+}$-TT Abs & $\begin{array}{c}\text { Vemurafenib and } \\
\text { Anti-225D9 }\end{array}$ \\
\hline 518A2 [BRAF(V600E)/CSPG4 $\left.{ }^{+}\right]$ & 6.4 & 6.6 & 6.7 & 6.8 \\
M14 [BRAF(V600E)/CSPG4] & 6.6 & 7.3 & 6.6 & 7.2 \\
\hline
\end{tabular}

even slightly increased and reached a cell index of 6.0 when compared to untreated cells. In contrast, vemurafenib together with anti-225D9+-TT Abs decreased the migration capacity of $518 \mathrm{~A} 2$ cells, reaching a cell index of 3.0 after $50 \mathrm{~h}$.

To test the invasive potential of 518A2 melanoma cells, a Transwell matrigel invasion assay was performed. In normoxic conditions, untreated 518A2 cells did not show any invasion capacity, reaching a cell index of 0.1 after $22 \mathrm{~h}$ (Fig. 4B). Vemurafenib or vemurafenib together with anti-225D9+-TT Abs increased the cell indices slightly to 0.85 and 1.4 , respectively. However, in hypoxic conditions the invasion capacity of untreated 518A2 melanoma cells increased and the cell index reached 1.4 after $22 \mathrm{~h}$. Surprisingly, when the cells were exposed to vemurafenib or vemurafenib and anti-225D9+-TT Abs, the effect was intensified with cell indices reaching 4.0 and 7.2 after $22 \mathrm{~h}$, respectively.
Anti-225D9+-TT Abs influence the metabolism in hypoxic, vemurafenib-treated melanoma cells. Untreated 518A2 cells showed a decrease in the extracellular $\mathrm{pH}$. After adding vemurafenib, anti-225D9 ${ }^{+}-\mathrm{TT}$ Abs or a combination of both, the extracellular $\mathrm{pH}$ decreased more slowly compared to untreated 518A2 cells (Fig. 5A and Table I). No change was observed in oxygen consumption between treated and untreated 518A2 cells (Fig. 5B). In contrast, no anti-225D9+-TT Abs-induced shift in extracellular $\mathrm{pH}$ was observed in the CSPG4 negative M14 melanoma cell line (Fig. 5A and Table I). A minor change for oxygen consumption from 2 to $4 \%$ was observed in this cell line in all treatment modalities (Fig. 5B).

Vemurafenib and anti-225D $9^{+}-T T$ Abs differently affect multiple signaling pathways in normoxic and hypoxic conditions. To understand the molecular heterogeneity in 
A
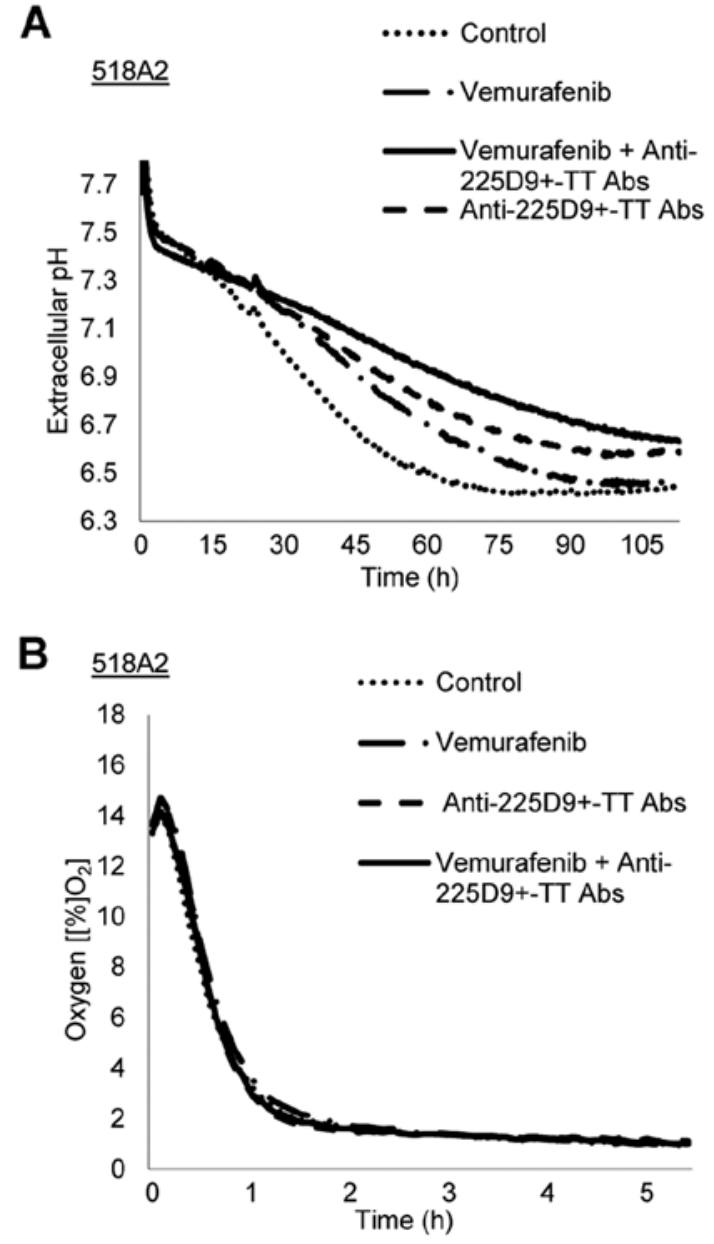
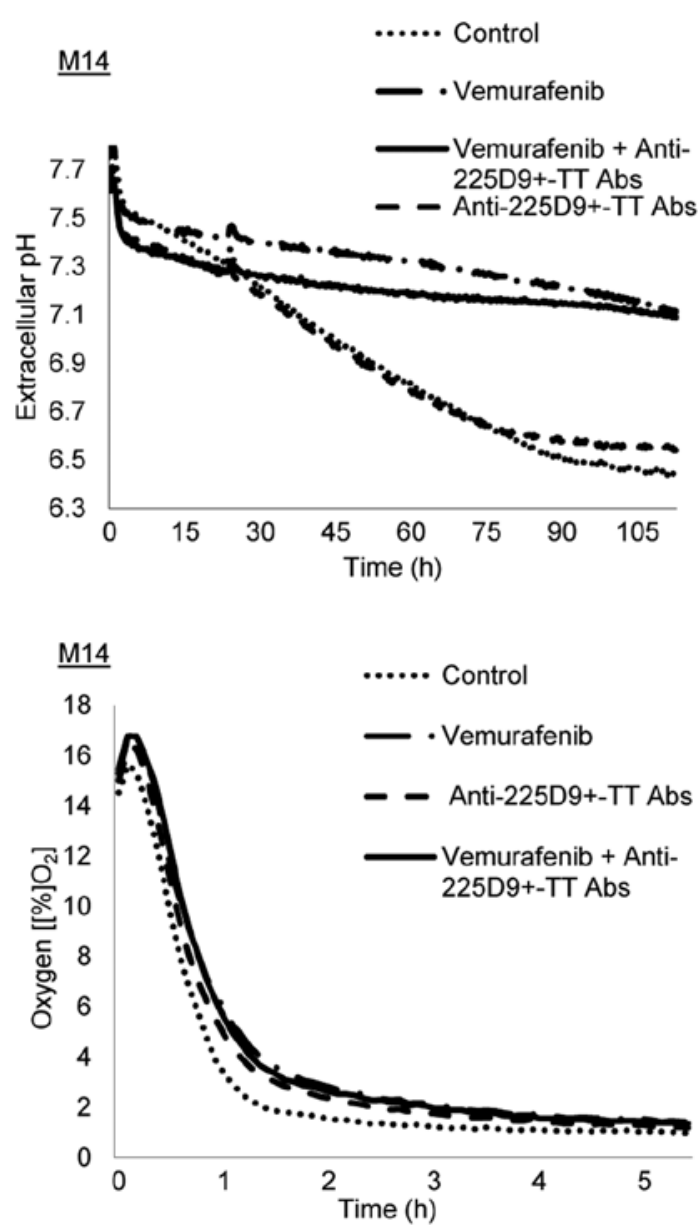

Figure 5. Anti-225D9+-TT Abs influence the metabolism in hypoxic, vemurafenib-treated melanoma cells. (A) Effect of vemurafenib and anti-225D9+-TT Abs on the extracellular $\mathrm{pH}$ regulation in hypoxic 518A2 melanoma cells [BRAF(V600E)/CSPG4 ${ }^{+}$] compared to M14 cells [BRAF(V600E)/CSPG4-]. (B) Effect of vemurafenib and anti-225D9+-TT Abs on the oxygen consumption in hypoxic 518A2 melanoma cells [BRAF(V600E)/CSPG4 $4^{+}$compared to M14 cells [BRAF(V600E)/CSPG4].

melanoma we compared the effects on signaling pathways important to cell growth, migration, invasion and survival in 518A2 melanoma cells after treatment with vemurafenib in normoxic and hypoxic conditions. In normoxic conditions, vemurafenib treatment of the melanoma cell line 518A2 resulted in a decreased protein expression level of pERK1/2 (Thr202/Tyr204) (Fig. 6B) and decreased expression levels of pFAK (Tyr397), FAK and AKT compared to untreated 518A2 cells (Fig. 6A). However, in hypoxic conditions untreated melanoma cells showed an increased protein expression of FAK and AKT compared to untreated cells in normoxia. The expression levels of PKC $\alpha$ and pAKT (Ser473) increased in vemurafenib-treated and untreated hypoxic 518A2 cells compared to normoxic cells (Fig. 6A).

To understand the molecular mechanisms underlying the modification of vemurafenib-mediated effects when combined with anti-225D9+-TT Abs, AKT and ERK signaling pathways were analyzed. The phosphorylation level of AKT (Ser473) increased in hypoxic conditions. However, pAKT (Ser473) slightly decreased after the treatment anti-225D9+-TT Abs and was decreased even more when vemurafenib was combined with anti-225D9+-TT Abs. No change was observed in the phosphorylation level of ERK1/2 (Thr202/Tyr204) (Fig. 6B).

\section{Discussion}

Melanoma is one of the most aggressive skin cancers. The poor efficiency of available therapies demands to find new therapeutic strategies to improve patient survival rates and to overcome resistance to currently used drugs $(8,34)$. Oncogenic mutations within the MAPK pathway are frequent in melanoma and targeting of MAPK signaling has yielded significant responses in a large number of patients that last for several months before relapsing (35).

One of the factors which contributes to melanoma progression is hypoxia through HIF-mediated molecular responses. HIF1 promotes the upregulation of genes which control a series of metabolic changes in tumor cells as well as increasing their invasive properties $(36,37)$. Thereby hypoxia significantly affects tumor phenotype and in many tumor types is associated with therapy resistance $(38,39)$. In melanoma, hypoxia is a microenvironmental stimulus that triggers a switch from a proliferative to an invasive cell phenotype that is less sensitive to therapies $(21,26)$. In our studies, hypoxia did not influence the proliferative capacity of 518A2 melanoma cells, whereas hypoxia enhanced the antiproliferative capacity of vemurafenib in a time-dependent manner (Fig. 1A). One 

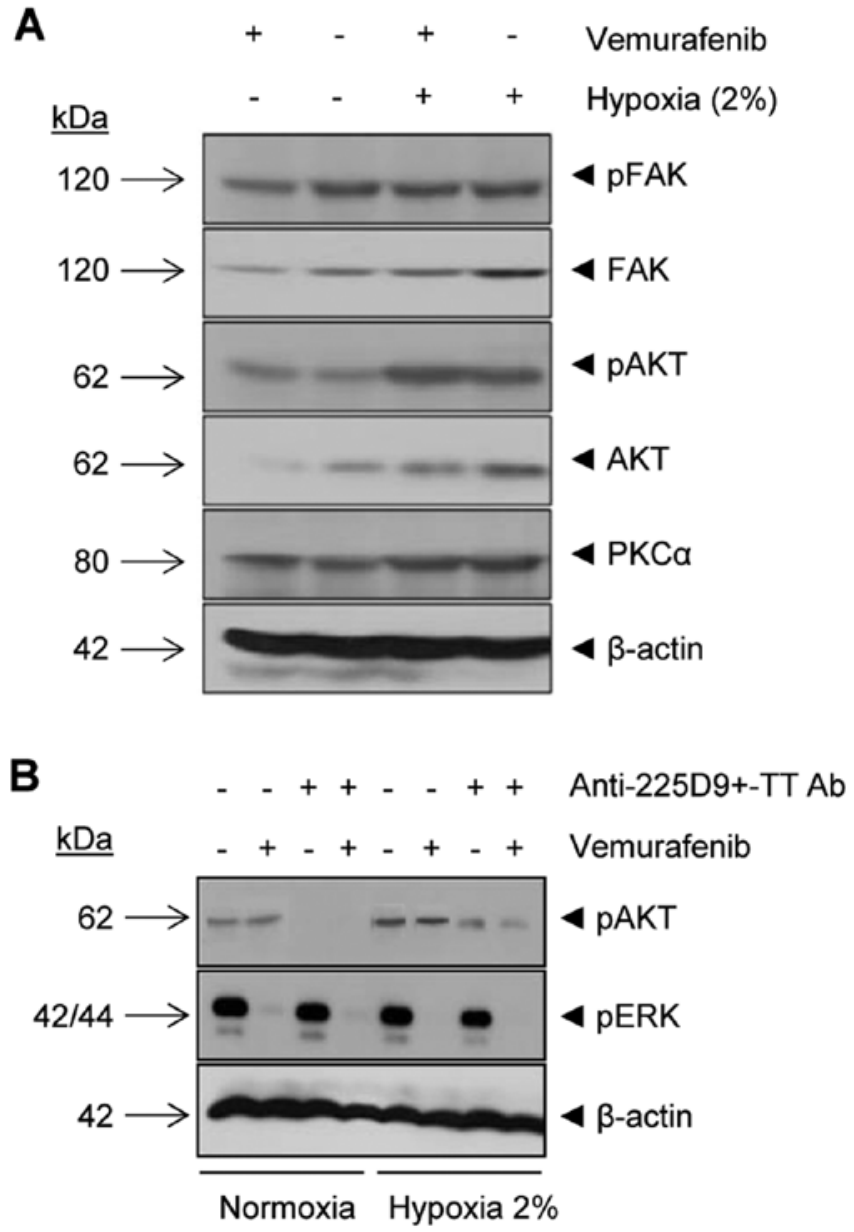

Figure 6. Hypoxia affects multiple signaling pathway proteins in 518A2 melanoma cells treated with vemurafenib and anti-225D9+-TT Abs. (A) Immunoblot analyses of proteins involved in PI3K, JAK-STAT and MAPK pathways under normoxic and hypoxic conditions after treatment with vemurafenib. (B) Western blotting of pAKT (Ser473) and pERK1/2 (Thr202/ Tyr204) in 518A2 cells treated with vemurafenib and anti-225D9+-TT Abs in normoxic and hypoxic conditions.

of the mediators of hypoxic responses in many cell types is the HIF1 $\alpha$ protein $(36,37)$. Vemurafenib markedly reduced the protein expression of this hypoxic marker in 518A2 melanoma cells and this downregulation also contributed to lower expression levels of CAIX (Fig. 1C). This was not reflected on the mRNA level of HIF1 $\alpha$, suggesting that the transcription of HIF1 $\alpha$ is not influenced by vemurafenib (Fig. 1B). Widmer et al (26) showed that knock-down of HIFla under hypoxic conditions decreased the invasion capacity of melanoma cells. We demonstrated that reduced HIF1 $\alpha$ expression was responsible for enhanced Transwell migration and invasion despite unchanged HIFl $\alpha$ mRNA levels (Figs. 1B, and 4). CAIX is a hypoxia-inducible, tumor-associated member of the human $\alpha$-CA family (40). CAIX shows only limited expression in normal tissues but its expression is highly elevated in various cancers such as colorectal and lung carcinomas $(41,42)$ and has not been shown in melanomas (43). To the best of our knowledge, we demonstrate for the first time CAIX expression and its downregulation by vemurafenib in 518A2 melanoma cells (Fig. 1C). We also observed CAIX staining in 518A2 xenografts in many nested tumor regions, preferable near necrotic areas co-localized with HIF1 $\alpha$ (Fig. 2).
Clinical evidence has already shown that melanoma regression is rarely complete after 6-9 months of therapy with vemurafenib (7). This is partly due to acquired resistance that leads to melanoma progression and a boost in aggressiveness of the disease (34) but also to the tumor microenvironment and the fact that melanomas are composed of heterogeneous zones containing proliferative and quiescent cells (44). A combined approach with vemurafenib and inhibitors targeting other signaling pathways determined to be abnormal in melanoma cells would be preferential. Therefore, we combined vemurafenib with antibodies directed against CSPG4 and evaluated their therapeutic potential in normoxic and hypoxic conditions. The CSPG4-specific mAb 225.28S was shown to inhibit the growth and recurrence of melanoma in mice grafted with CSPG4-expressing human melanoma cell lines (16) and the antibodies induced by a mimotope vaccine (anti-225D9+-TT Abs) directed against CSPG4 were capable of inhibiting melanoma cell growth in a SCID mouse model $(18,19)$. In line with these data we found that anti-225D9 ${ }^{+}$-TT Abs were effective in normoxic conditions against proliferative cells and reduced the growth of human melanoma $518 \mathrm{~A} 2$ cells by $30 \%$ (Fig. 3A). Here we show that the combination of vemurafenib and anti-225D9+-TT Abs could reduce the growth of 518A2 cells by additional $30 \%$ (Fig. 3B). Comparable results were achieved with the CSPG4specific mAb 225.28 (17).

This finding provided the rationale for the strategy described in this report that combined vemurafenib with antibodies directed against CSPG4 as a means of enhancing the magnitude of the BRAF inhibitor responses that could also influence migration and invasion. There are varying results regarding the effect of MAPK pathway inhibition on invasion and migration in the literature $(44,45)$. We tracked 518A2 cells in the presence of vemurafenib and anti-225D9+-TT Abs for long periods of time within a hypoxic microenvironment and obtained a longitudinal track for invasion and migration. We showed that Transwell migration of 518A2 cells toward a chemoattractant was markedly increased in hypoxic conditions and even more after the treatment with vemurafenib. This effect was partly blocked by anti-225D9+-TT Abs (Fig. 4A) reflecting the role of CSPG4 in cell migration (12). However, the addition of anti225D9+-TT Abs markedly enhanced the invasive capacity of vemurafenib-treated, hypoxic 518A2 cells (Fig. 4B).

Our data imply that hypoxia is important in determining the effect of targeting CSPG4 on cell migration and invasion. Since hypoxia is associated with changes in the extracellular $\mathrm{pH}$ and related to the acidification of the tumor microenvironment that promotes cancer cell invasion (46), we were able to show that vemurafenib increased the extracellular $\mathrm{pH}$ in 518A2 cells, an effect that was diminished by the addition of anti-225D9+-TT Abs (Fig. 5A and Table I). This was not reflected in the oxygenation levels (Fig. 5B) underlining the role of CSPG4 irrespective of oxygenation in tumor microenvironment and showing its potential as a therapeutic target. In a next step we focused on the capacity of vemurafenib and anti-225D9+-TT Ab to block multiple signaling pathways in hypoxic and normoxic conditions. Kumar et al (30) showed that the BRAF inhibitor sorafenib inhibited ERK phosphorylation and suggested that some of the effect of this compound was mediated through HIF1 $\alpha$ inhibition. Hypoxia per se influenced 
multiple signaling pathways such as pAKT (Ser473), AKT and PKC $\alpha$ (Fig. 6A). In this study we found that the expression of pAKT (Ser473) decreased after treatment with vemurafenib and anti-225D9+-TT Abs in hypoxic conditions (Fig. 6B) confirming that hypoxia affected the response to vemurafenib and anti-225D9+-TT Abs in 518A2 melanoma cells.

In this study we showed that targeting CSPG4 in melanoma cells enhanced antiproliferative effects of vemurafenib in normoxic conditions and reduced the migratory capacity in hypoxic conditions. Hypoxia strongly influenced the response to vemurafenib treatment in melanoma cells which switched to a more invasive and aggressive phenotype. Therefore, therapeutic efforts will have to consider that the microenvironment of melanoma cells has an impact on tumor progression.

\section{Acknowledgements}

This project is a part of the EU Marie Curie Initial Training Network (ITN) Biomedical engineering for cancer and brain disease diagnosis and therapy development (EngCaBra). Project no. PITN-GA-2010-264417.

\section{References}

1. MacKie RM, Hauschild A and Eggermont AMM: Epidemiology of invasive cutaneous melanoma. Ann Oncol 20 (Suppl 6): vi1-vi7, 2009.

2. Linos E, Swetter SM, Cockburn MG, Colditz GA and Clarke CA: Increasing burden of melanoma in the United States. J Invest Dermatol 129: 1666-1674, 2009.

3. Testori A, Rutkowski P, Marsden J, Bastholt L, Chiarion-Sileni V, Hauschild A and Eggermont AM: Surgery and radiotherapy in the treatment of cutaneous melanoma. Ann Oncol 20 (Suppl 6): vi22-vi29, 2009.

4. Kee D and McArthur G: Targeted therapies for cutaneous melanoma. Hematol Oncol Clin North Am 28: 491-505, 2014.

5. Gogas H, Polyzos A and Kirkwood J: Immunotherapy for advanced melanoma: Fulfilling the promise. Cancer Treat Rev 39: 879-885, 2013

6. Chapman PB, Hauschild A, Robert C, Haanen JB, Ascierto P, Larkin J, Dummer R, Garbe C, Testori A, Maio M, et al; BRIM-3 Study Group: Improved survival with vemurafenib in melanoma with BRAF V600E mutation. N Engl J Med 364: 2507-2516, 2011.

7. Flaherty KT, Puzanov I, Kim KB, Ribas A, McArthur GA, Sosman JA, O'Dwyer PJ, Lee RJ, Grippo JF, Nolop K, et al: Inhibition of mutated, activated BRAF in metastatic melanoma N Engl J Med 363: 809-819, 2010.

8. Fedorenko IV, Paraiso KH and Smalley KS: Acquired and intrinsic BRAF inhibitor resistance in BRAF V600E mutant melanoma. Biochem Pharmacol 82: 201-209, 2011.

9. Sun C, Wang L, Huang S, Heynen GJ, Prahallad A, Robert C, Haanen J, Blank C, Wesseling J, Willems SM, et al: Reversible and adaptive resistance to BRAF(V600E) inhibition in melanoma. Nature 508: 118-122, 2014.

10. Kugel CH III and Aplin AE: Adaptive resistance to RAF inhibitors in melanoma. Pigment Cell Melanoma Res 27: 1032-1038, 2014.

11. Kudchadkar R, Paraiso KH and Smalley KS: Targeting mutant BRAF in melanoma: Current status and future development of combination therapy strategies. Cancer J 18: 124-131, 2012.

12. Price MA, Colvin Wanshura LE, Yang J, Carlson J, Xiang B, Li G, Ferrone S, Dudek AZ, Turley EA and McCarthy JB: CSPG4, a potential therapeutic target, facilitates malignant progression of melanoma. Pigment Cell Melanoma Res 24: $1148-1157,2011$

13. Mittelman A, Chen ZJ, Liu CC, Hirai S and Ferrone S: Kinetics of the immune response and regression of metastatic lesions following development of humoral anti-high molecular weightmelanoma associated antigen immunity in three patients with advanced malignant melanoma immunized with mouse antiidiotypic monoclonal antibody MK2-23. Cancer Res 54: 415-421, 1994.
14. Ferris RL, Jaffee EM and Ferrone S: Tumor antigen-targeted, monoclonal antibody-based immunotherapy: Clinical response, cellular immunity, and immunoescape. J Clin Oncol 28: 4390-4399, 2010

15. Wang X, Osada T, Wang Y, Yu L, Sakakura K, Katayama A, McCarthy JB, Brufsky A, Chivukula M, Khoury T, et al: CSPG4 protein as a new target for the antibody-based immunotherapy of triple-negative breast cancer. J Natl Cancer Inst 102: 1496-1512, 2010.

16. Hafner C, Breiteneder H, Ferrone S, Thallinger C, Wagner S, Schmidt WM, Jasinska J, Kundi M, Wolff K, Zielinski CC, et al: Suppression of human melanoma tumor growth in SCID mice by a human high molecular weight-melanoma associated antigen (HMW-MAA) specific monoclonal antibody. Int J Cancer 114: 426-432, 2005.

17. Yu L, Favoino E, Wang Y, Ma Y, Deng X and Wang X: The CSPG4-specific monoclonal antibody enhances and prolongs the effects of the BRAF inhibitor in melanoma cells. Immunol Res 50: 294-302, 2011

18. Wagner S, Hafner C, Allwardt D, Jasinska J, Ferrone S, Zielinski CC, Scheiner O, Wiedermann U, Pehamberger $\mathrm{H}$ and Breiteneder H: Vaccination with a human high molecular weight melanoma-associated antigen mimotope induces a humoral response inhibiting melanoma cell growth in vitro. J Immunol 174: 976-982, 2005 .

19. Wagner S, Krepler C, Allwardt D, Latzka J, Strommer S, Scheiner O, Pehamberger H, Wiedermann U, Hafner C and Breiteneder H: Reduction of human melanoma tumor growth in severe combined immunodeficient mice by passive transfer of antibodies induced by a high molecular weight melanomaassociated antigen mimotope vaccine. Clin Cancer Res 14: 8178-8183, 2008 .

20. Walsh JC, Lebedev A, Aten E, Madsen K, Marciano L and Kolb HC: The clinical importance of assessing tumor hypoxia: Relationship of tumor hypoxia to prognosis and therapeutic opportunities. Antioxid Redox Signal 21: 1516-1554, 2014.

21. O'Connell MP, Marchbank K, Webster MR, Valiga AA, Kaur A, Vultur A, Li L, Herlyn M, Villanueva J, Liu Q, et al: Hypoxia induces phenotypic plasticity and therapy resistance in melanoma via the tyrosine kinase receptors ROR1 and ROR2. Cancer Discov 3: 1378-1393, 2013.

22. Vergis R, Corbishley CM, Norman AR, Bartlett J, Jhavar S, Borre M, Heeboll S, Horwich A, Huddart R, Khoo V, et al: Intrinsic markers of tumour hypoxia and angiogenesis in localised prostate cancer and outcome of radical treatment: A retrospective analysis of two randomised radiotherapy trials and one surgical cohort study. Lancet Oncol 9: 342-351, 2008.

23. Mujcic H, Hill RP, Koritzinsky M and Wouters BG: Hypoxia signaling and the metastatic phenotype. Curr Mol Med 14: 565-579, 2014.

24. Ghislin S, Deshayes F, Middendorp S, Boggetto N and Alcaide-Loridan C: PHF19 and Akt control the switch between proliferative and invasive states in melanoma. Cell Cycle 11: 1634-1645, 2012.

25. Erler JT, Bennewith KL, Nicolau M, Dornhöfer N, Kong C, Le QT, Chi JT, Jeffrey SS and Giaccia AJ: Lysyl oxidase is essential for hypoxia-induced metastasis. Nature 440: 1222-1226, 2006.

26. Widmer DS, Hoek KS, Cheng PF, Eichhoff OM, Biedermann T, Raaijmakers MI, Hemmi S, Dummer R and Levesque MP: Hypoxia contributes to melanoma heterogeneity by triggering HIF1 $\alpha$-dependent phenotype switching. J Invest Dermatol 133: 2436-2443, 2013

27. Hanna SC, Krishnan B, Bailey ST, Moschos SJ, Kuan PF, Shimamura T, Osborne LD, Siegel MB, Duncan LM, O'Brien ET III, et al: HIF $1 \alpha$ and HIF $2 \alpha$ independently activate SRC to promote melanoma metastases. J Clin Invest 123: 2078-2093, 2013

28. Mouriaux F, Sanschagrin F, Diorio C, Landreville S, Comoz F, Petit E, Bernaudin M, Rousseau AP, Bergeron D and Morcos M: Increased HIF-1 $\alpha$ expression correlates with cell proliferation and vascular markers CD31 and VEGF-A in uveal melanoma. Invest Ophthalmol Vis Sci 55: 1277-1283, 2014.

29. Ditte P,Dequiedt F, Svastova E, Hulikova A, Ohradanova-Repic A, Zatovicova M, Csaderova L, Kopacek J, Supuran CT, Pastorekova S, et al: Phosphorylation of carbonic anhydrase IX controls its ability to mediate extracellular acidification in hypoxic tumors. Cancer Res 71: 7558-7567, 2011.

30. Kumar SM, Yu H, Edwards R, Chen L, Kazianis S, Brafford P, Acs G, Herlyn M and Xu X: Mutant V600E BRAF increases hypoxia inducible factor-1alpha expression in melanoma. Cancer Res 67: 3177-3184, 2007. 
31. Limame R, Wouters A, Pauwels B, Fransen E, Peeters M, Lardon F, De Wever O and Pauwels P: Comparative analysis of dynamic cell viability, migration and invasion assessments by novel real-time technology and classic endpoint assays. PLoS One 7: e46536, 2012.

32. Chiche J, Brahimi-Horn MC and Pouysségur J: Tumour hypoxia induces a metabolic shift causing acidosis: A common feature in cancer. J Cell Mol Med 14: 771-794, 2010.

33. Pastoreková S, Závadová Z, Kostál M, Babusíková O and Závada J: A novel quasi-viral agent, MaTu, is a two-component system. Virology 187: 620-626, 1992.

34. Trunzer K, Pavlick AC, Schuchter L, Gonzalez R, McArthur GA, Hutson TE, Moschos SJ, Flaherty KT, Kim KB, Weber JS, et al: Pharmacodynamic effects and mechanisms of resistance to vemurafenib in patients with metastatic melanoma. J Clin Oncol 31: 1767-1774, 2013

35. Cheng Y, Zhang G and Li G: Targeting MAPK pathway in melanoma therapy. Cancer Metastasis Rev 32: 567-584, 2013.

36. Irwin DC, McCord JM, Nozik-Grayck E, Beckly G, Foreman B, Sullivan T, White M, T Crossno J Jr, Bailey D, Flores SC, et al: A potential role for reactive oxygen species and the HIF-1alpha-VEGF pathway in hypoxia-induced pulmonary vascular leak. Free Radic Biol Med 47: 55-61, 2009.

37. Dewhirst MW: Relationships between cycling hypoxia, HIF-1, angiogenesis and oxidative stress. Radiat Res 172: 653-665, 2009.

38. Liao J, Qian F, Tchabo N, Mhawech-Fauceglia P, Beck A, Qian Z, Wang X, Huss WJ, Lele SB, Morrison CD, et al: Ovarian cancer spheroid cells with stem cell-like properties contribute to tumor generation, metastasis and chemotherapy resistance through hypoxia-resistant metabolism. PLoS One 9: e84941, 2014.

39. Azab AK, Hu J, Quang P, Azab F, Pitsillides C, Awwad R, Thompson B, Maiso P, Sun JD, Hart CP, et al: Hypoxia promotes dissemination of multiple myeloma through acquisition of epithelial to mesenchymal transition-like features. Blood 119 : 5782-5794, 2012.
40. McDonald PC, Winum JY, Supuran CT and Dedhar S: Recent developments in targeting carbonic anhydrase IX for cancer therapeutics. Oncotarget 3: 84-97, 2012.

41. Ivanov S, Liao SY, Ivanova A, Danilkovitch-Miagkova A, Tarasova N, Weirich G, Merrill MJ, Proescholdt MA, Oldfield EH, Lee J, et al: Expression of hypoxia-inducible cellsurface transmembrane carbonic anhydrases in human cancer. Am J Pathol 158: 905-919, 2001.

42. Chrastina A, Závada J, Parkkila S, Kaluz S, Kaluzová M, Rajcáni J, Pastorek J and Pastoreková S: Biodistribution and pharmacokinetics of 125I-labeled monoclonal antibody M75 specific for carbonic anhydrase IX, an intrinsic marker of hypoxia, in nude mice xenografted with human colorectal carcinoma. Int J Cancer 105: 873-881, 2003.

43. Syrjänen L, Luukkaala T, Leppilampi M, Kallioinen M, Pastorekova S, Pastorek J, Waheed A, Sly WS, Parkkila S and Karttunen T: Expression of cancer-related carbonic anhydrases IX and XII in normal skin and skin neoplasms. APMIS 122: 880-889, 2014.

44. Haass NK, Beaumont KA, Hill DS, Anfosso A, Mrass P, Munoz MA, Kinjyo I and Weninger W: Real-time cell cycle imaging during melanoma growth, invasion, and drug response. Pigment Cell Melanoma Res 27: 764-776, 2014.

45. Vultur A, Villanueva J, Krepler C, Rajan G, Chen Q, Xiao M, Li L, Gimotty PA, Wilson M, Hayden J, et al: MEK inhibition affects STAT3 signaling and invasion in human melanoma cell lines. Oncogene 33: 1850-1861, 2014.

46. Sedlakova O, Svastova E, Takacova M, Kopacek J, Pastorek J and Pastorekova S: Carbonic anhydrase IX, a hypoxia-induced catalytic component of the $\mathrm{pH}$ regulating machinery in tumors. Front Physiol 4: 400, 2014. 